\title{
Intra-Abdominal Pressure Measurements in Neurocritical Patients
}

\author{
Xuxu Xu ${ }^{1}$, Zhenghui He${ }^{1}$, Yingchi Shan ${ }^{2}$, Qing Mao ${ }^{1}$, Junfeng Feng ${ }^{1}$, Guoyi Gao ${ }^{2}$ \\ ${ }^{1}$ Department of Neurosurgery, Renji Hospital, Shanghai Jiaotong University School of Medicine ${ }^{2}$ Department of Neurosurgery, Shanghai General Hospital, \\ Shanghai Jiaotong University School of Medicine \\ * These authors contributed equally
}

Corresponding Author

Guoyi Gao

gao3@sina.com

\section{Date Published}

May 11, 2021

\section{Citation}

Xu, X., He, Z., Shan, Y., Mao, Q., Feng, J., Gao, G. Intra-Abdominal Pressure Measurements in Neurocritical Patients. J. Vis. Exp. (171), e62557, doi:10.3791/62557 (2021)

DOI

$10.3791 / 62557$
URL

jove.com/video/62557

\section{Abstract}

Intra-abdominal pressure (IAP) is increasingly being recognized as an indispensable and significant physiological parameter in intensive care units (ICU). IAP has been measured in a variety of ways with the development of many techniques in recent years. The level of intra-abdominal pressure under normal conditions is generally equal to or less than $12 \mathrm{mmHg}$. Accordingly, abdominal hypertension (IAH) is defined as two consecutive IAP measurements higher than $12 \mathrm{mmHg}$ within 4-6 $\mathrm{h}$. When IAH deteriorates further with IAP higher than $20 \mathrm{mmHg}$ along with organ dysfunction and/ or failure, this clinical manifestation can be diagnosed as abdominal compartment syndrome (ACS). IAH and ACS are associated with gastrointestinal ischemia, acute renal failure, and lung injury, leading to severe morbidity and mortality. Elevated IAP and IAH may affect the cerebral venous return and outflow of the cerebrospinal fluid by increasing the intrathoracic pressure (ITP), ultimately leading to increased intracranial pressure (ICP). Therefore, it is essential to monitor IAP in critically ill patients. The reproducibility and accuracy of intra-bladder pressure (IBP) measurements in previous studies need to be further improved, although the indirect measurement of IAP is now a widely used technique.

To address these limitations, we recently used a set of IAP monitoring systems with advantages of convenience, continuous monitoring, digital visualization, and long-term IAP recording and data storage in critically ill patients. This IAP monitoring system can detect intra-abdominal hypertension and potentially analyze clinical status in real time. The recorded IAP data and other physiological indicators, such as intracranial pressure, can be further used for correlation analysis to guide treatment and predict a patient's possible prognosis. 


\section{Introduction}

The pressure in the abdominal cavity is known as the intra-abdominal pressure (IAP). Accordingly, abdominal hypertension $(\mathrm{IAH})$ is defined as two consecutive IAP measurements higher than $12 \mathrm{mmHg}$ within 4-6 $\mathrm{h}^{1}$. When IAH deteriorates further, with IAP higher than $20 \mathrm{mmHg}$ and organ dysfunction or failure, the clinical manifestation can be diagnosed as abdominal compartment syndrome (ACS). $\mathrm{IAH}$ and ACS are associated with gastrointestinal ischemia, acute renal failure, and lung injury, leading to severe morbidity and mortality ${ }^{1,2,3,4}$. Increased IAP can also lead to diaphragmatic elevation, which increases intrathoracic pressure to reduce pulmonary compliance and increases central venous pressure ${ }^{5,6}$. Cerebral venous return in the jugular system and outflow of the cerebrospinal fluid may be disturbed by excessive intra-thoracic pressure ${ }^{7,8}$, leading to intracranial congestion and intracranial hypertension, which can further cause brain dysfunction and affect the prognosis $^{9}, 10,11,12,13,14$. Another theoretical approach is that increased IAP will cause the blood from the sacral venous plexus and vertebral veins to return to the spinal canal, thereby causing intraspinal vein congestion and eventually venous blood flowing into the brain, leading to an increased intracranial pressure ${ }^{8}$. Currently, IAP is measured by both direct and indirect methods ${ }^{15,16,17,18}$. The intermittent recording of intra-bladder pressure using an indwelling bladder catheter is the most common and widely accepted method $^{19,20,21,22}$. This convenient and quick method still relies on the periodical pressure measurements and is usually labor-intensive and time-consuming ${ }^{23}$.

To solve these difficulties, we have recently adopted a set of IAP monitoring systems with advantages of convenience, continuous monitoring, digital visualization for IAP measurement, and long-term IAP data recording and storage for neurocritical patients. The system is developed for the bladder pressure measurement, and its built-in pressure sensor measures the fluid pressure in the urinary catheter to obtain intra-abdominal pressure. This IAP monitoring system can be used to confirm intra-abdominal hypertension, to analyze the current clinical status, and to predict the possible prognosis of neurosurgical ICU patients. Compared with previous intra-abdominal pressure measurements, this intra-abdominal pressure monitoring system has certain advantages: the system is portable and easy to use; IAP data can be collected and stored in real-time every minute; multiple parameters (i.e., IAP, urinary output, and urinary flow rate) can be measured, recorded, and visualized; and the monitoring is long-term, continuous, and less susceptible to urinary tract infections than previous methods.

Therefore, the purpose of this paper is to present a detailed approach of using digitalized monitoring systems to record IAP for patients with severe traumatic brain injury (TBI) or intracranial hemorrhage $(\mathrm{ICH})$.

\section{Protocol}

This protocol has been approved by the Institutional Review Board of Renji Hospital affiliated with the Shanghai Jiao Tong University School of Medicine.

\section{Patient inclusion and exclusion criteria}

1. Include patients with Glasgow coma scale $(\mathrm{GCS}) \leq 8$, 18-85 years of age who have been diagnosed with TBI or $\mathrm{ICH}$. The diagnosis of traumatic brain injury or cerebral hemorrhage was performed by clinicians using clinical manifestations and radiographic features.

2. Exclude patients who cannot tolerate catheterization. Also, exclude patients who have urinary tract infections, 
gastrointestinal system diseases, coagulopathy, and immune metabolic diseases.

NOTE: In the present study, enrolled subjects were all adults, and there was no sex bias. If patients experience pain during catheterization, inject $2 \mathrm{~mL}$ of $1 \%$ lidocaine hydrochloride into the patient's lower urinary tract to relieve the discomfort.

3. Lay the patient in a supine position with genitals exposed and use iodophor to thoroughly disinfect the patient's perineum and urethral orifice three times.

4. Lubricate the Foley catheter (16 Fr) in the catheter bag and insert the whole catheter into the patient's urethra. Inject $20 \mathrm{~mL}$ of sterile normal saline into the balloon of the Foley catheter. Then slowly pull out the Foley catheter until the urine flows out of the catheter.

NOTE: If the patient needs surgery on admission, perform catheterization in the operating room. If not, perform catheterization in the neurologic intensive care unit.

\section{Preparations and connections}

NOTE: The IAP monitoring system consists of three components: disposable pressure sensors, a urodynamic monitor, and intra-abdominal pressure and urine volume data management software (see Table of Materials).

1. Connect the urinary catheter to the collection bag with a sensor and fix the midline of the tee fittings at the same level as the patient's axilla's midline plane (Figure 1).

2. Check that the entire tube is unobstructed to ensure that it can be filled with urine.

3. Install the sensor on the urine collection bag onto the IAP monitoring device Figure 2).
NOTE: The IAP monitoring device has a unique card slot for the sensor, and the sensor can be inserted into the slot to work.

4. Hang the urine collection bag below the IAP monitoring device.

NOTE: When the urine collection bag reaches $2000 \mathrm{~mL}$, the IAP monitoring device will sound an alarm. Empty the urine collection bag to continue monitoring.

5. Press the Power button to turn on the IAP monitoring device.

6. Enter the timing mode, set the mode parameters as required, and enter the standard display interface to determine the closed valve.

NOTE: Timing mode sets a certain time interval to open the valve to release urine in the catheter. For this experiment, the time to open the valve was set to every $0.5 \mathrm{~h}$ and the duration was set to $3 \mathrm{~min}$ because, in this way, the IAP can be measured without affecting the patient's urination. The valve is located in the sensor and is usually closed in the mode setting.

7. Rotate the tee fitting, point the OFF end to the patient's urinary duct's direction, and unscrew the Luer joint sealing cap.

NOTE: This step is similar to the invasive arterial pressure zeroing. There is a knob on the tee to rotate and close the catheter segment and loosen the sealing cap at the air end of the connection. The sensor on the equipment contacts the air to obtain the value of atmospheric pressure.

8. Long press the return key for $3 \mathrm{~s}$ to enter the sensor setting interface, press OK to reset to zero, and press OK after "reset to zero completed" is displayed. 
9. Screw back the sealing cap and rotate the tee so that the OFF points towards the Ruhr cap's direction.

10. After IBP in the standard display interface becomes positive and stable, the IBP displayed is the IAP value of the patient (Figure 3).

NOTE: If the displayed value is negative or greater than $30 \mathrm{mmHg}$, it means that it was not zeroed correctly and needs to be reset again.

\section{Recording and storing data of IAP}

NOTE: An intra-abdominal pressure and urine volume data management software is used to record and store IAP data. Ensure that the software is correctly installed on the work computer.

1. Connect the IAP monitor and a computer via a wireless network on a router.

2. Open the intra-abdominal pressure and urine volume data management software on the laptop with the key inserted and enter the password to log in.

3. Wait for a few seconds until the software runs automatically.

4. 3.4.Click the Medical Record button and enter the patient's information, including the age, ID, bed number, gender, diagnosis.

5. Click the Save button and wait $10 \mathrm{~s}$ to start data collection and storing. Figure 4 shows real-time data displayed in the software.

6. Click the Layout Settings button to display the real-time monitoring of multiple patients as required (max=64). Adjust the window interface to see multipatient monitoring and adjust the timeline to see the requirements' details, including the daily urine volume, daily changes in intra-abdominal pressure trend, demonstrated in Figure 5.

7. Click the Trend Review button to see the detailed parameter changes,including the recorded parameter values and the trend chart for the duration.

NOTE: Since the pressure sensor is valid for one week, ensure the patient's urine collection bag is replaced with a regular one. The data recorded by the software can be used for further analysis. It can be combined with other physiological data such as correlation analysis with intracranial pressure monitored at the same time to determine the effect of intra-abdominal pressure on intracranial pressure. To facilitate the calculation, 0.5 $\mathrm{h}$ mean IAP and $0.5 \mathrm{~h}$ mean ICP were selected to perform correlation analysis and plot the scatterplot using a statistical software.

\section{Representative Results}

This new type of continuous IAP monitoring system was used in eight neurosurgical ICU patients (7 males) on admission. Figure 4 shows real-time data obtained from the software. The IAP in this figure indicates that the patient is in a state of intraabdominal hypertension. The physician can adjust the window interface to see multi-patient monitoring and adjust the timeline to see the requirements' details, including the daily urine volume, daily changes in intra-abdominal pressure trend, demonstrated in Figure 5. Six of the patients (75\%) had suffered from intracranial hemorrhage $(\mathrm{ICH})$, and two of them $(25 \%)$ had a traumatic brain injury (TBI) (Table 1). The mean IAP of five patients was less than $12 \mathrm{mmHg}$. We observed that the IAP of two patients was between 12 to $20 \mathrm{mmHg}$, and one patient had IAP over $20 \mathrm{mmHg}$ (Figure 6). Three of the eight patients were also monitored for ICP, and the correlation between ICP and IAP was significant (Figure 7). The total monitoring time is 7 days per patient. Clinical diagnoses and 


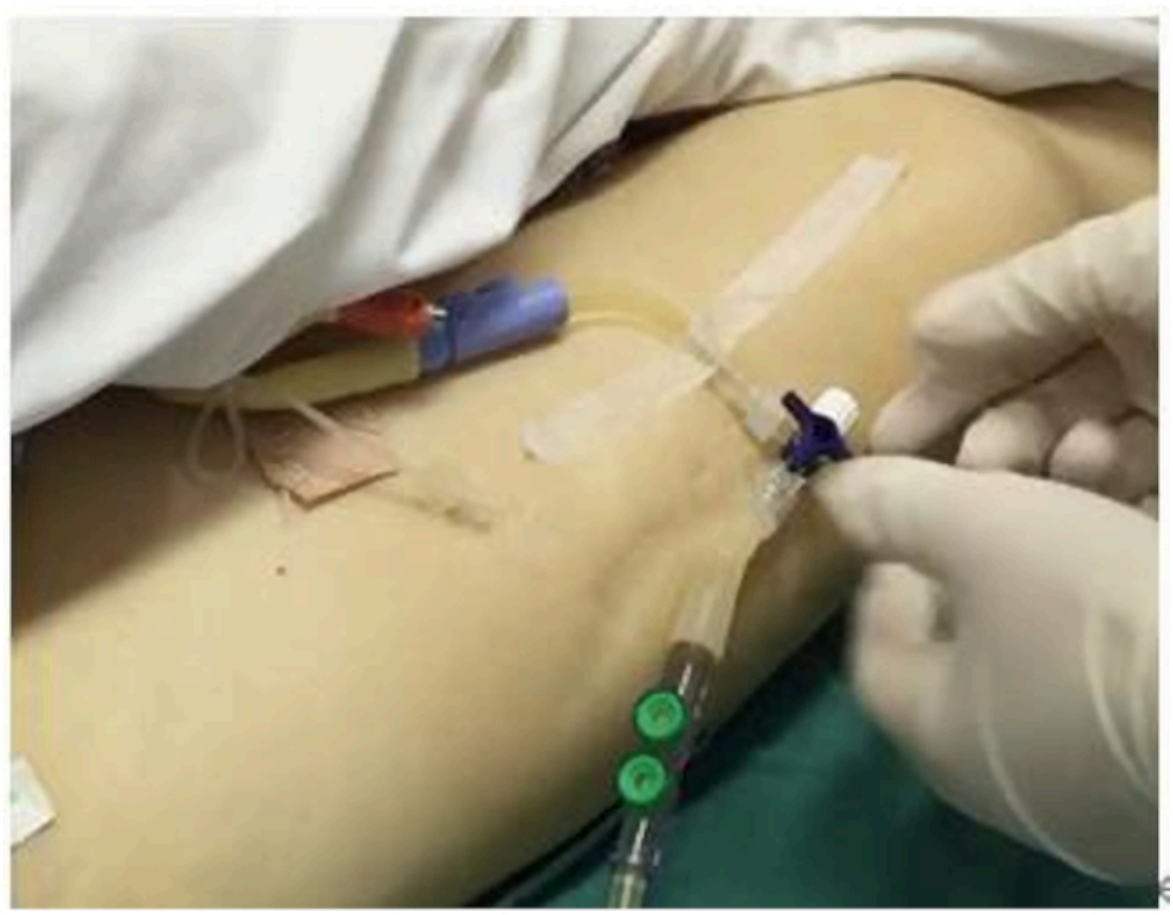

Figure 1: IAP sensor connection. The patient needs to be catheterized before the connection setup. This figure shows the connection and fixation of the sensor. Please click here to view a larger version of this figure. 


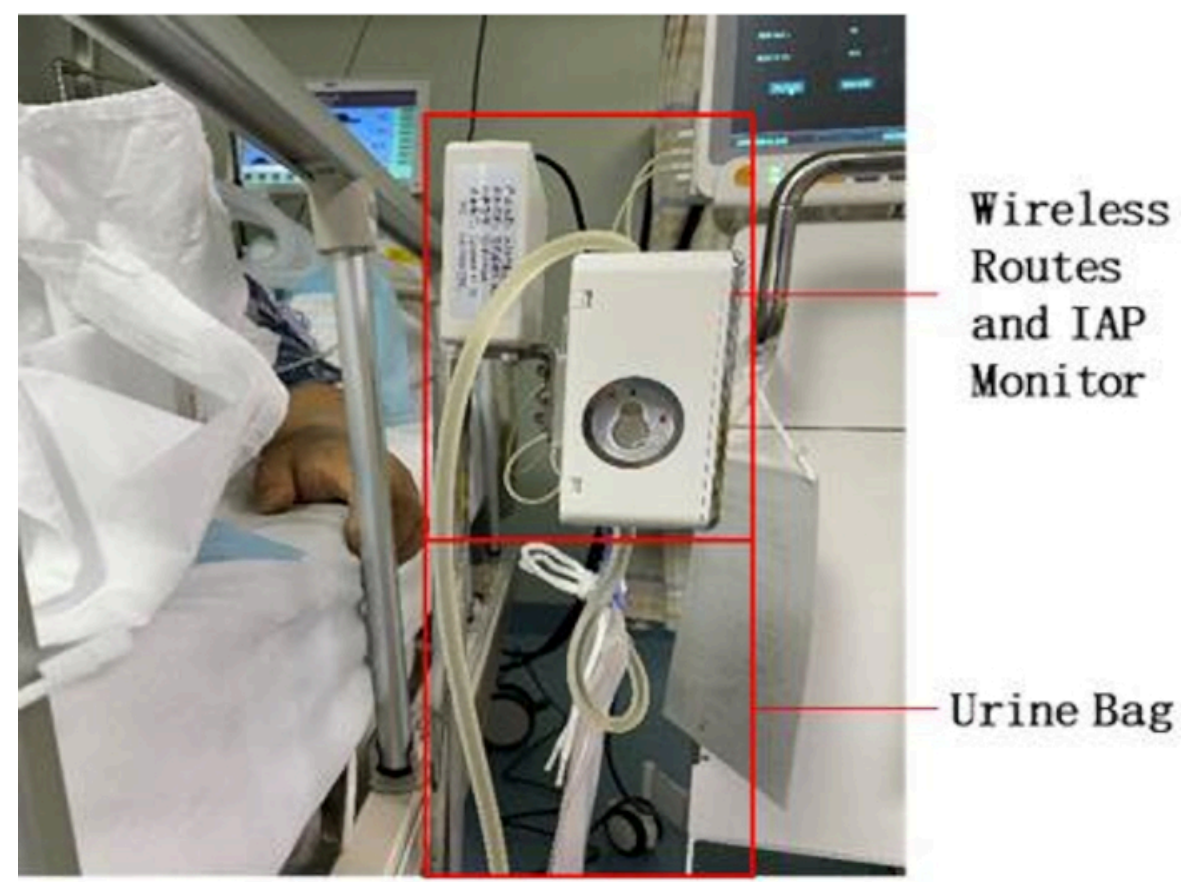

Figure 2: Bedside equipment placement. Install the valve on the urine collection bag on the equipment and place it next to the bed. Connect the power supply. Please click here to view a larger version of this figure.

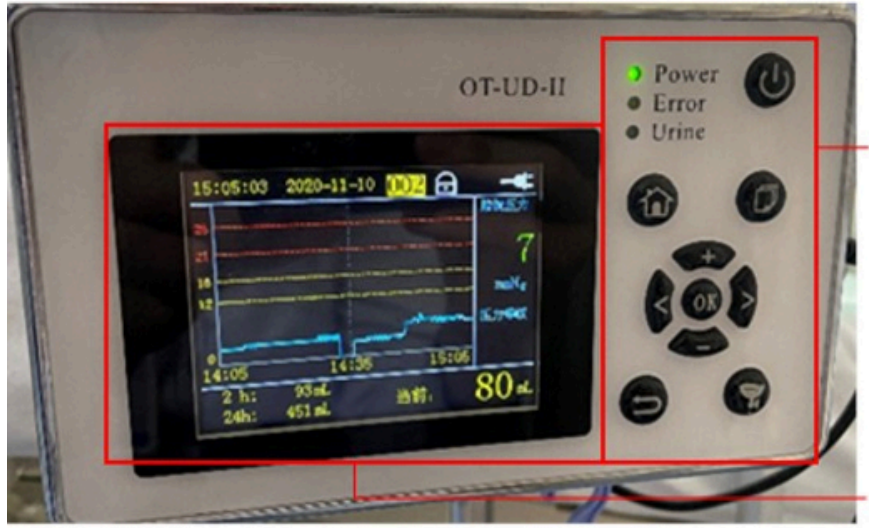

Exhibited

Light and

Press-button

IAP Display

Interface

Figure 3: Screen of IAP monitoring device. The middle of the screen shows the current IAP waveform, number, and pressure rating. Time and urine output is displayed at the top and bottom of the screen, respectively. The right side is a variety of function keys, and the upper right side is the power supply. Please click here to view a larger version of this figure. 


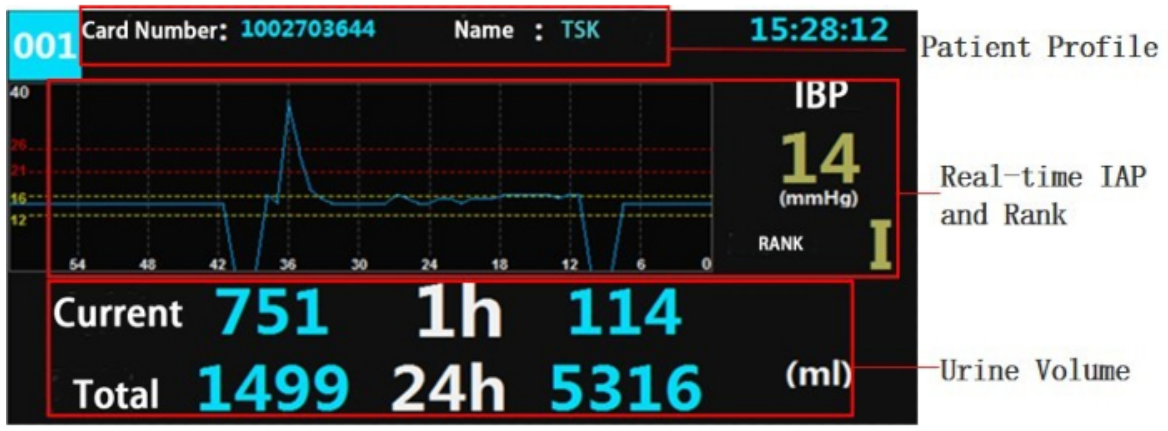

Figure 4: Screen of the monitoring device. The upper screen shows the patient's information, including bed number, hospital number, and present time. The middle screen shows that the real-time IAP is $14 \mathrm{mmHg}$ and the pressure rank is grade I. The lower screen shows that the patient's current urine volume is $751 \mathrm{~mL}$, the average hourly urine volume is 114 $\mathrm{mL}$, the urine volume in the collection bag is $1499 \mathrm{~mL}$, and the total urine volume within $24 \mathrm{~h}$ is $5316 \mathrm{~mL}$. Please click here to view a larger version of this figure.

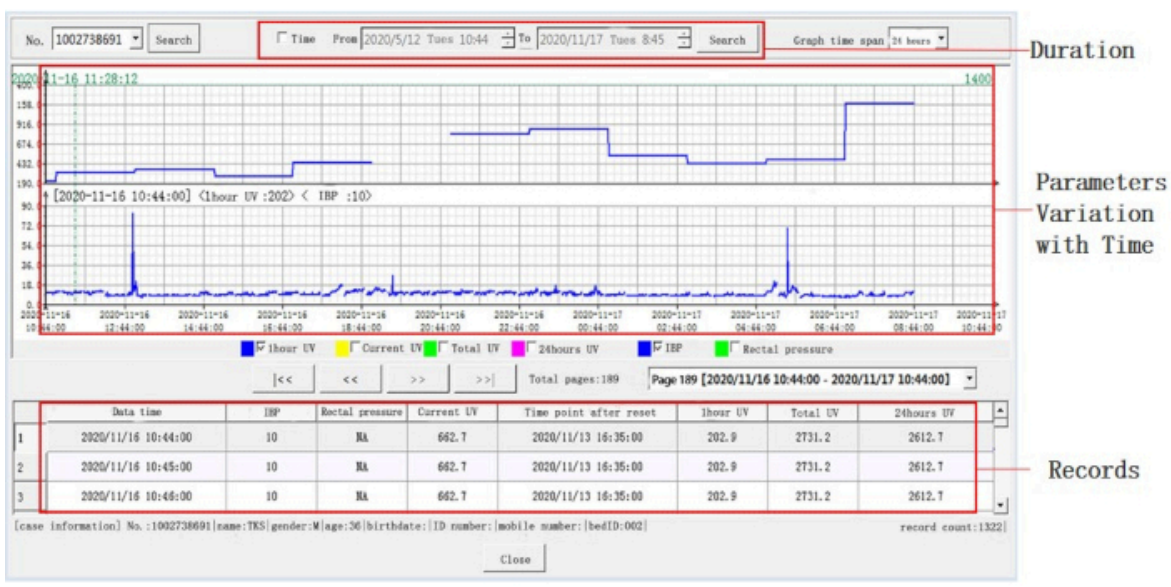

Figure 5: Detailed information on representative data. More details were displayed after adjusting the timeline. The timeline is on the $\mathrm{X}$-axis, and the separate parameters are on the $\mathrm{Y}$-axis, shown in the middle window. The default duration is $24 \mathrm{~h}$. Blue represents hourly urine volume or IBP. Intra-abdominal pressure per minute, hourly urine volume, cumulative urine volume, and $24 \mathrm{~h}$ urine volume can also be reviewed through the lower window. Please click here to view a larger version of this figure. 


\begin{tabular}{|c|c|}
\hline Characteristics & Value, $n(\%)$ \\
\hline Number of patients & 8 \\
\hline \multicolumn{2}{|l|}{ Gender, $n(\%)$} \\
\hline Male & $7(87.5)$ \\
\hline Age, mean $\pm S D$ (years) & $52.4 \pm 18.2$ \\
\hline \multicolumn{2}{|l|}{ Diagnosis, $n$ (\%) } \\
\hline$T B I$ & $2(25.0)$ \\
\hline $\mathrm{ICH}$ & $6(75.0)$ \\
\hline GCS (admission), mean $\pm S D$ & $6.1 \pm 1.6$ \\
\hline GCS, mean $\pm S D$ & $7.0 \pm 2.0$ \\
\hline GOS, mean $\pm S D$ & $2.25 \pm 0.5$ \\
\hline Length of stay, mean $\pm S D$ & $37.9 \pm 16.8$ \\
\hline Operation, $n(\%)$ & $6(75.0)$ \\
\hline \multicolumn{2}{|l|}{ Comorbidity, $n$ (\%) } \\
\hline hypertension & $5(62.5)$ \\
\hline others & $3(37.5)$ \\
\hline
\end{tabular}

Table 1. Clinical characteristics of patients. 


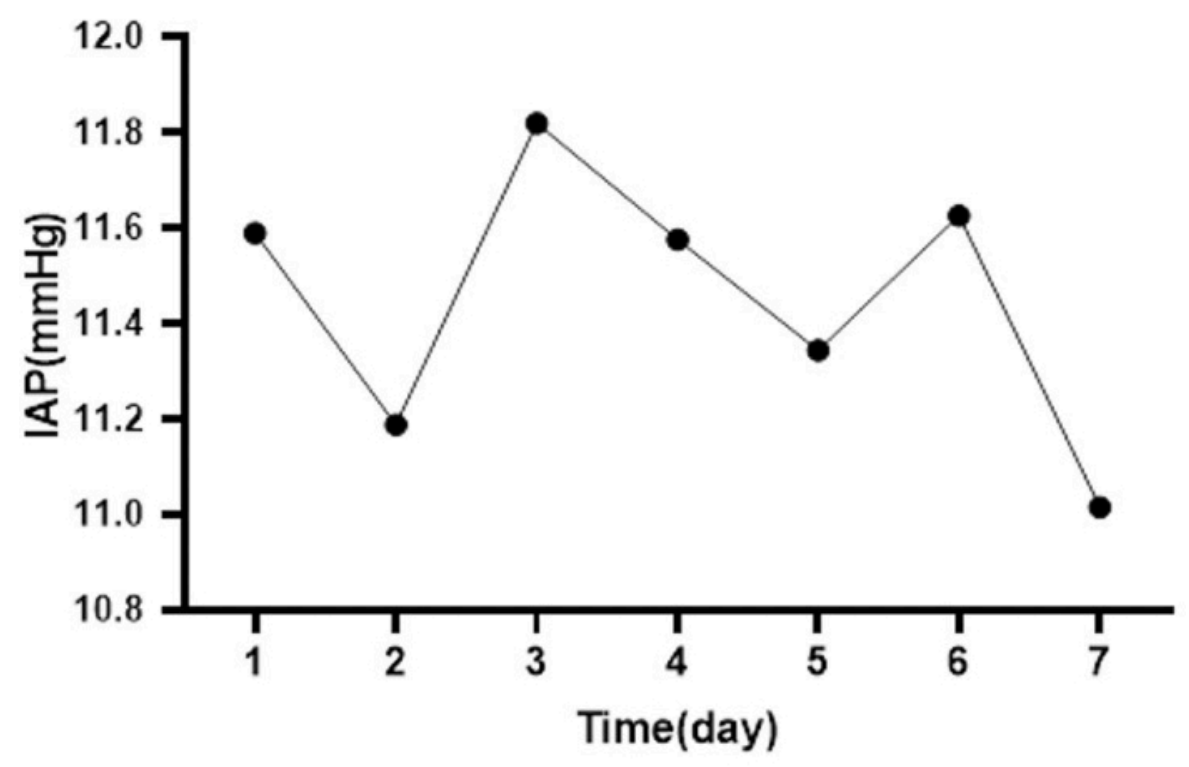

Figure 6: Mean IAP changes for all patients during the week. This picture shows the change in average daily IAP for eight patients, one of whom had the highest mean IAP (mean IAP=20.75 mmHg, on the 3rd day), and the other had the lowest mean IAP (mean IAP=0.06 $\mathrm{mmHg}$, on the 2 nd day). Please click here to view a larger version of this figure. 


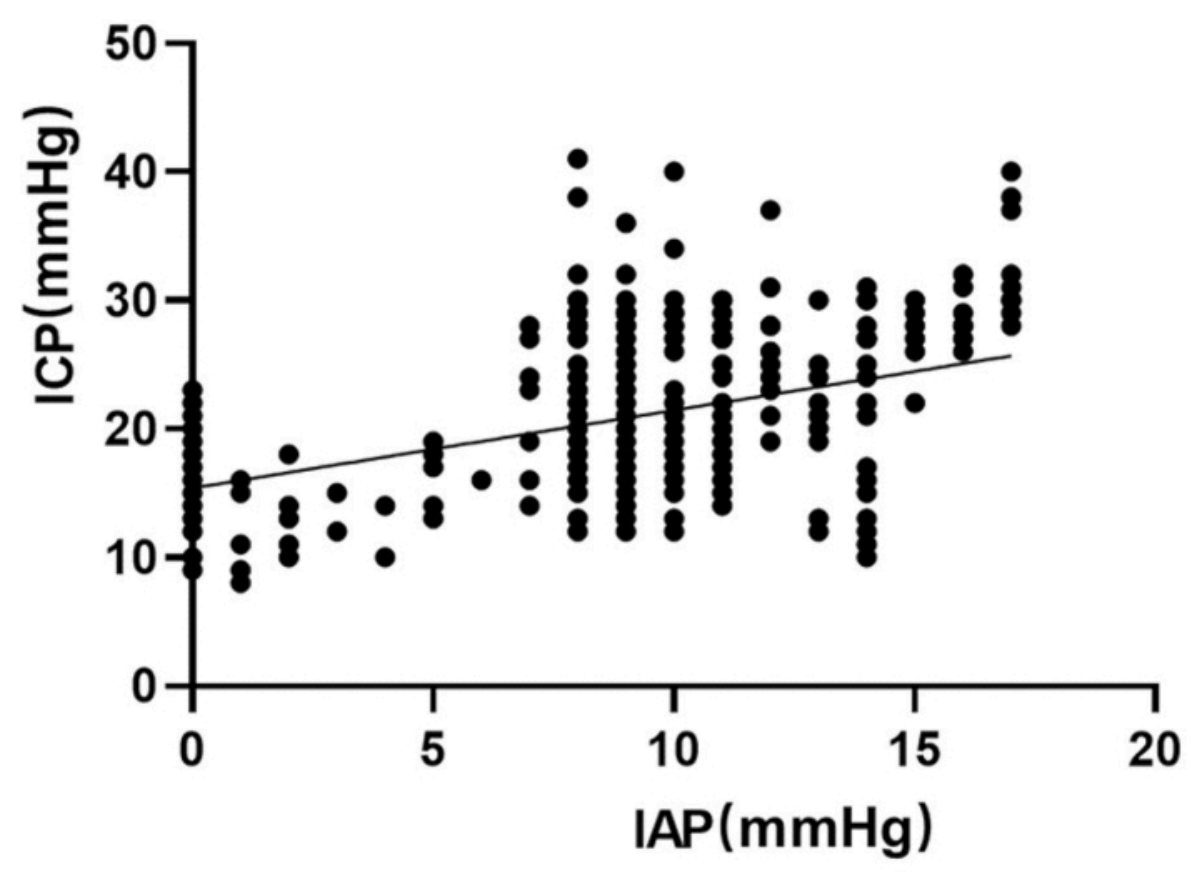

Figure 7: Scatter plots of IAP and ICP. ICP values were also monitored in three of the eight patients, and the correlation between ICP and IAP was significant $(r=0.47, p<0.01, \alpha=0.05)$. Please click here to view a larger version of this figure.

\section{Discussion}

This brief article's primary purpose is to introduce continuous monitoring and data storage systems for critical patients in the neurosurgical (ICU). This system can digitally and continuously monitor IAP, thereby providing the possibility of a wider time window of data storage, review, and analysis. The variation of the IAP, especially the IAP increment, always indicates the change of physical status of intraabdominal organs, such as acute gastrointestinal injury under neurocritical circumstances. Further, the increased IAP may consequently influence intra-thoracal pressure. The affected central venous pressure positively impacts the intracranial status, such as intracranial pressure ${ }^{11,12}$. The abdominal perfusion pressure evaluation, which is related closely to the cerebral perfusion pressure, provides a convenient method to manage cerebral blood supply if appropriately applied $^{10,13}$. For the reasons above, detection of intraabdominal hypertension exhibits the potential to analyze the current clinical status, and, hopefully, predicts the patients' prognosis in the neurosurgical ICU.

Over the last three decades, IAP's concerns have been rediscovered, better articulated and have achieved numerous clinical significance $11,24,25$. The ideal IAP measurement method was tried over 150 years ago and has since evolved into direct and indirect measurement methods. The former is measured surgically by placing a Veress needle or intraperitoneal catheters in the abdominal cavity and connecting it to pressure transducers $26,27,28$. The latter measures the pressure in the abdominal organs, including the stomach, bladder, uterus, and rectum $29,30,31$. Among 
them, intra-bladder pressure (IBP) is the most accepted IAP measurement method by researchers because it is practical, feasible, and non-invasive features ${ }^{20,21,32}$. It can be obtained by connecting a catheter to a three-way tube and injecting $25 \mathrm{~mL}$ of sterile normal saline into the catheter lumen $^{2}$. The physician used the above method routinely to measure the intra-abdominal pressure of patients in the neurological ICU. However, a great deal of time and effort is wasted on data with limited timeliness because it cannot be continuously monitored and recorded. The IAP monitoring system resolves the problem of intermittent bladder pressure measurement. However, all parts need to be connected correctly and zeroed correctly according to the above protocol. Otherwise, the incorrectly measured IAP will affect the diagnosis and treatment of the disease state. The IAP monitoring system has been monitored in 8 patients with critical neurological conditions. Although no system failures or errors have been reported, we recommend seeking the assistance of the system maintenance engineer in the event of a problem. Moreover, some minor defects need to be further resolved, such as the $2,000 \mathrm{~mL}$ maximum volume of the urine collection bag and one week validity of the pressure transducers.

In brief, the measuring system introduced in the manuscript demonstrates the advantages of accurate data measurement, long-term monitoring, data digitalization, storage, and visualization. Also, the autonomic operation, after properly applied, solves the labor burden in ICU settings. The intraabdominal pressure monitoring system may become a routine procedure in neurointensive care units in the future, but further clinical evaluation on the relationship of IAP with clinical manifestation is needed.

\section{Disclosures}

National nature science foundation of china (81971699).

\section{Acknowledgments}

We want to thank all the colleagues in the Neurological Intensive Care Unit for their work.

\section{References}

1. Pereira, B. M. Abdominal compartment syndrome and intra-abdominal hypertension. Current Opinion in Critical Care. 25 (6), 688-696 (2019).

2. Sadeghi, M. et al. Abdominal Compartment Syndrome in Critically III Patient. Open Access Macedonian Journal of Medical Sciences. 7 (7), 1097-1102 (2019).

3. Maluso, P., Olson, J., Sarani, B. Abdominal compartment hypertension and abdominal compartment syndrome. Critical Care cClinics. 32 (2), 213-222 (2016).

4. Oda, J. et al. Resuscitation fluid volume and abdominal compartment syndrome in patients with major burns. Burns. 32 (2), 151-154 (2006).

5. Regli, A., Pelosi, P., Malbrain, M. Ventilation in patients with intra-abdominal hypertension: What every critical care physician needs to know. Annals of Intensive Care. 9 (1), 52 (2019).

6. Rosenthal R. J. et al. Effects of hyperventilation and hypoventilation on $\mathrm{PaCO}_{2}$ and intracranial pressure during acute elevations of intraabdominal pressure with $\mathrm{CO}_{2}$ pneumoperitoneum: Large animal observations. Journal of the American College of Surgeons. 187 (1), 32-38 (1998). 
7. Wilson, M. H. Monro-Kellie 2.0: The dynamic vascular and venous pathophysiological components of intracranial pressure. Journal of Cerebral Blood Flow and Metabolism: Official Journal of the International Society of Cerebral Blood Flow and Metabolism,. 36 (8), 1338-1350 (2016).

8. Depauw, P. et al. The significance of intra-abdominal pressure in neurosurgery and neurological diseases: A narrative review and a conceptual proposal. Acta Neurochirurgica(Wien). 161 (5), 855-864 (2019).

9. De Laet, I., Citerio, G., Malbrain, M. L. The influence of intra-abdominal hypertension on the central nervous system: Current insights and clinical recommendations, is it all in the head? Acta Clinica Belgica. 62 Suppl 1, 89-97 (2007).

10. Deeren D. H, Dits H, Malbrain M. L. N. GCorrelation between intra-abdominal and intracranial pressure in nontraumatic brain injury. Intensive Care Medicine. 31 (11), 1577-1581 (2005).

11. Cheatham, M. L. Abdominal compartment syndrome: pathophysiology and definitions. Scandinavian Journal of Trauma, Resuscitation and Emergency Medicine. 17, 10 (2009).

12. Ben-Haim, M., Mandeli, J., Friedman, R. L., Rosenthal, R. J. Mechanisms of systemic hypertension during acute elevation of intraabdominal pressure. The Journal of Surgical Research. 91 (2), 101-105 (2000).

13. Marinis A et al. Ischemia as a possible effect of increased intra-abdominal pressure on central nervous system cytokines, lactate and perfusion pressures. Critical Care. 14 (2), R31 (2010).
14. Scalea, T. M. et al. Increased intra-abdominal, intrathoracic, and intracranial pressure after severe brain injury: Multiple compartment syndrome. Journal of Trauma. 62 (3), 647-656; discussion 656 (2007).

15. Schachtrupp, A. et al. Evaluation of two novel methods for the direct and continuous measurement of the intraabdominal pressure in a porcine model. Intensive Care Medicine. 29 (9), 1605-1608 (2003).

16. Malbrain, M. L. Different techniques to measure intraabdominal pressure (IAP): Time for a critical re-appraisal. Intensive Care Medicine. 30 (3), 357-371 (2004).

17. Polistena, A. et al. Local radiotherapy of exposed murine small bowel: apoptosis and inflammation. BioMed Central Surgery. 8, 1 (2008).

18. van Waes, O. J. et al. A single-lumen central venous catheter for continuous and direct intra-abdominal pressure measurement. European Journal of Trauma and Emergency Surgery : Official Publication of the European Trauma Society. 35 (6), 532-537 (2009).

19. Kron, I. L., Harman, P. K., Nolan, S. P. The measurement of intra-abdominal pressure as a criterion for abdominal re-exploration. Annals of Surgery. 199 (1), 28-30 (1984).

20. Iberti, T. J., Lieber, C. E., Benjamin, E. Determination of intra-abdominal pressure using a transurethral bladder catheter: Clinical validation of the technique. Anesthesiology. 70 (1), 47-50 (1989).

21. Fusco, M. A., Martin, R. S., Chang, M. C. Estimation of intra-abdominal pressure by bladder pressure measurement: Validity and methodology. Journal of Trauma. 50 (2), 297-302 (2001).

22. Al-Abassi, A. A., Al Saadi, A. S., Ahmed, F. Is intrabladder pressure measurement a reliable indicator 
for raised intra-abdominal pressure? A prospective comparative study. BMC Anesthesiology. 18 (1), 69 (2018).

23. De Waele, J. J., De Laet, I., Malbrain, M. L. Rational intraabdominal pressure monitoring: how to do it? Acta Clinica Belgica. 62 Suppl 1, 16-25 (2007).

24. Japiassú, A. M. et al. Measurement of intra-abdominal pressure in the intensive care unit: the opinion of the critical care physicians. Revista Brasileira de Terapia Intensiva. 19 (2), 186-191 (2007).

25. Ravishankar, N., Hunter, J. Measurement of intraabdominal pressure in intensive care units in the United Kingdom: A national postal questionnaire study. British journal of anaesthesia. 94 (6), 763-766 (2005).

26. Schachtrupp, A. et al. Evaluation of two novel methods for the direct and continuous measurement of the intraabdominal pressure in a porcine model. Intensive Care Medicine. 29 (9), 1605-1608 (2003).

27. Risin $E$ et al. New technique of direct intra-abdominal pressure measurement. Asian Journal of Surgery. 29 (4), 247-250 (2006).

28. Risin, E., Kessel, B., Ashkenazi, I., Lieberman, N., Alfici, R. A new technique of direct intra-abdominal pressure measurement: a preliminary study. American Journal of Surgery. 191 (2), 235-237 (2006).

29. Wauters, J. et al. A novel method (CiMON) for continuous intra-abdominal pressure monitoring: Pilot test in a pig model. Critical Care Research and Practice. 2012, 181563 (2012).

30. Staelens, A. S. et al. Intra-abdominal pressure measurements in term pregnancy and postpartum: an observational study. PLoS One. 9 (8), e104782 (2014).
31. Rosenbluth, E. M., Johnson, P. J., Hitchcock, R. W., Nygaard, I. E. Development and testing of a vaginal pressure sensor to measure intra-abdominal pressure in women. Neurourology and Urodynamics. 29 (4), 532-535 (2010).

32. Van Stappen, J. et al. Validation of a novel method for measuring intra-abdominal pressure and gastric residual volume in critically ill patients. Anaesthesiology Intensive Therapy. 46 (4), 245-254 (2014) 